\title{
Characterization of the S-Phase-Specific Transcription Regulatory Elements in a DNA Replication-Independent Testis-Specific H2B (TH2B) Histone Gene
}

\author{
INHWAN HWANG, † KYU LIM, \\ Department of Biochemistry, University of North Carolina, Chapel Hill, North Carolina 27599-7260
}

Received 8 May 1989/Accepted 12 October 1989

\begin{abstract}
The testis-specific H2B histone (TH2B) gene is expressed in pachytene spermatocytes of meiotic prophase I during rat spermatogenesis. The TH2B RNA and histones are not synthesized in any other tissues, and the synthesis is independent of DNA replication. However, the cloned TH2B gene has two DNA sequence elements which stimulate transcription of the cloned gene in an S-phase-dependent manner when introduced into somatic cells. The factors interacting with the two elements, CCAAT at -127 base pairs and octamer ATTTGCAT at -93 base pairs, interact with each other to bring about a maximum stimulation of S-phase-dependent transcription. The level of CCAAT and octamer-binding proteins is unchanged during the cell cycle, and the S-phase-dependent transcription of TH2B and endogenous mouse H2B genes does not require synthesis of new proteins during the $S$ phase. Cell cycle-specific posttranslational modification of regulatory proteins may be responsible for the S-phase-dependent transcription of $H 2 B$ histone genes. The biological significance of the presence of S-phase-specific transcription regulatory elements in the DNA replication-independent and tissue-specific TH2B gene is not known.
\end{abstract}

Synthesis of somatic histones is coupled with DNA replication and controlled by a 2- to 3-fold increase of gene transcription and $>20$-fold increase in the stabilization of histone mRNA during the $S$ phase $(20,39,41)$. The mechanism of S-phase-dependent expression of histone genes has been investigated by gene transfer experiments with cloned histone genes. The S-phase-specific regulatory elements (SRE) for several histone genes have been characterized ( 2 , $8,25,27,33,42,46)$. However, none of the genes seem to have any common sequence element for the $S$-phase-specific regulation. This suggests that the $S$-phase-specific transcription of different histone genes may be regulated by different mechanisms or by different trans-acting factors. Recently, a nuclear protein factor which interacts with the human $\mathrm{H} 2 \mathrm{~B}$ SRE containing an octamer motif (ATTTGCAT) has been purified from HeLa cell nuclear extracts and shown to stimulate transcription of the $\mathrm{H} 2 \mathrm{~B}$ gene in vitro (12).

We have cloned a cDNA for a meiosis-specific H2B histone variant (TH2B) from rat testis (23). In situ cytohybridization showed that the expression of TH2B gene is confined to pachytene spermatocytes of meiotic prophase I (23), in which there is very little DNA synthesis except for DNA repair activity (29). Also, in contrast to somatic histones, synthesis of TH2B and other spermatogenic cellspecific histone variants is unaffected by inhibitors of DNA synthesis $(3,28)$. Therefore, expression of the TH2B gene appears to be cell type specific and independent of DNA replication. Surprisingly, however, the cloned TH2B gene is regulated in a cell-cycle-dependent manner as somatic histone genes when introduced into several somatic cell lines grown in culture (21; I. Hwang, unpublished results). This suggests that the TH2B gene contains SRE. The reason for

\footnotetext{
* Corresponding author.

$\dagger$ Present address: Department of Molecular Biology, Massachusetts General Hospital, Boston, MA 02144.

$\ddagger$ Permanent address: Chungnam University School of Medicine, Daejun, Korea.
}

the presence of SRE in the TH2B gene is not clear. Since the gene is not expressed except in pachytene spermatocytes, the SRE are most probably repressed in somatic cells. In pachytene spermatocytes, the SRE could be either repressed or involved in the cell-type-specific expression of TH2B gene. To gain insight into the role of the SRE in the regulation of TH2B genes during spermatogenesis, we have first characterized the DNA sequence elements involved in the S-phase-dependent transcription of the cloned TH2B gene in somatic cells.

We report that the CCAAT sequence element at -127 base pairs (bp) and the adjacent octamer element at $-93 \mathrm{bp}$ from the transcription initiation site play key roles in the S-phase-dependent transcription as well as in the overall stimulation of transcription of the cloned TH2B gene. The nuclear protein factors interacting with the two elements appear to interact with each other to bring about a maximal stimulation of gene transcription in the $S$ phase. Unlike other genes, stimulation of TH2B and somatic mouse $\mathrm{H} 2 \mathrm{~B}$ gene expression in the $S$ phase does not require synthesis of new proteins, and the level of nuclear proteins interacting with the $\mathrm{TH} 2 \mathrm{~B}$ regulatory region is not altered during the cell cycle. Cell-cycle-specific posttranslational modification of regulatory proteins may be responsible for the $S$-phasedependent expression of histone genes. The TH2B regulatory region has very similar sequence organization to a human somatic $\mathrm{H} 2 \mathrm{~B}$ gene (27). However, the mechanism of the S-phase-dependent transcription of the cloned TH2B gene appears to be different from that of the human somatic H2B gene (27).

\section{MATERIALS AND METHODS}

Site-directed mutagenesis. To introduce mutations into the octamer motif and CCAAT elements, we synthesized oligonucleotides containing desired base changes and used them as primers for synthesis of the complementary strand of the $5^{\prime}$ upstream region of the $\mathrm{TH} 2 \mathrm{~B}$ gene in single-stranded M13mp19 DNA. The detailed protocol for the oligonucle- 
otide-directed mutagenesis was described previously (26). The mutations were confirmed by DNA sequencing by the dideoxynucleotide method of Sanger et al. (37).

Construction of insertion mutants. For insertion of different lengths of DNA between the CCAAT at $-127 \mathrm{bp}$ and octamer elements, different sizes of DNA fragments were ligated to the EcoRI site between the CCAAT and octamer elements to produce half and integral turns of DNA helix.

Transfection of DNA into mouse embryo fibroblast C3H 10T1/2 cells and synchronization. The transient S-phasedependent expression of the genes introduced into the cells was studied by using a transient expression assay as described previously (21). Briefly, a mixture of $6 \mu \mathrm{g}$ of a test DNA and $5 \mu \mathrm{g}$ of pRSV $\beta$-gal (11) was added to mouse embryo fibroblast $\mathrm{C} 3 \mathrm{H} 10 \mathrm{~T} 1 / 2$ cells as $\mathrm{DNA}-\mathrm{CaPO}_{4}$ coprecipitates (15). The cells were washed 12 to $14 \mathrm{~h}$ after addition of the coprecipitates and incubated with aphidicolin (2 $\mu \mathrm{g} / \mathrm{ml}$ ) for $24 \mathrm{~h} \mathrm{(7).} \mathrm{To} \mathrm{release} \mathrm{the} \mathrm{cells} \mathrm{from} \mathrm{the} \mathrm{aphidicolin}$ block, we rinsed them with Hanks balanced salt solution and incubated them with a fresh medium without aphidicolin for an appropriate length of time. Cell extracts were prepared by brief sonication of the cells and centrifugation in a microcentrifuge (15), and $\beta$-galactosidase activity was determined as described previously (1). The amounts of extracts containing the same $\beta$-galactosidase activity were used to assay chloramphenicol acetyltransferase (CAT) activity $(15,17)$ to correct for differences in transfection efficiency among different culture dishes. The radioactive spots of acetylated chloramphenicol were scraped from thin-layer plates and counted in a scintillation counter.

Nuclear extracts. Nuclear extracts were prepared by the method of Dignam et al. (9), with the following modifications. To the salt extract of nuclei was added 0.1 vol of $4 \mathrm{M}$ $\left(\mathrm{NH}_{4}\right)_{2} \mathrm{SO}_{4}$, and the mixture was stirred for $30 \mathrm{~min}$ at $4^{\circ} \mathrm{C}$ and centrifuged for $1 \mathrm{~h}$ at $35,000 \mathrm{rpm}$ in a Sorvall T-865.1 rotor. Additional ammonium sulfate was added slowly to the supernatant $(0.25 \mathrm{~g} / \mathrm{ml})$, and the mixture was stirred for 30 min and centrifuged at $15,000 \mathrm{rpm}$ in a Sorvall SA600 rotor. The pellet was dissolved in a small volume of dialysis buffer (0.1 M KCl, $15 \mathrm{mM} N$-2-hydroxyethylpiperazine- $N^{\prime}-2$ ethanesulfonic acid [HEPES; pH 7.9], $20 \%$ glycerol, $2 \mathrm{mM}$ $\mathrm{MgCl}_{2}, 1 \mathrm{mM}$ dithiothreitol, $0.2 \mathrm{mM}$ EDTA, $0.5 \mathrm{mM}$ phenylmethylsulfonyl fluoride) and dialyzed against the same buffer for $5 \mathrm{~h}$. After removal of insoluble material, small aliquots were quick frozen in a dry ice-ethanol bath and stored at $-80^{\circ} \mathrm{C}$. For S-phase and non-S-phase nuclear extracts, semi- or fully confluent cells were replated at low density $\left(2 \times 10^{4}\right.$ cells per $\left.\mathrm{cm}^{2}\right)$, incubated in basal Eagle medium containing $10 \%$ heat-inactivated fetal bovine serum and aphidicolin for $24 \mathrm{~h}$ (non-S phase), and then released from the block for 2 to $3 \mathrm{~h}$ (S phase). The cycloheximidetreated S-phase nuclear extracts were prepared from the cells treated for 2 to $3 \mathrm{~h}$ with $20 \mu \mathrm{g}$ of cycloheximide per $\mathrm{ml}$ during the release of cells from the aphidicolin block. The concentration of proteins in nuclear extracts was determined by reading $A_{230}$ and $A_{260}$ as described previously (22).

DNA mobility shift assay. DNA probes were prepared by end labeling with $\left[{ }^{32} \mathrm{P}\right] \mathrm{ATP}(7,000 \mathrm{Ci} / \mathrm{mmol}$; Du Pont, NEN Research Products) in the presence of T4 polynucleotide kinase. For DNA mobility shift assays $(13,14,40)$, labeled DNA probes $\left(10^{4} \mathrm{cpm}\right)$ were mixed with $3 \mu$ l of nuclear extracts (protein concentration, $1.5 \mu \mathrm{g} / \mu \mathrm{l}$ ) in a $10-\mu$ l reaction volume containing $60 \mathrm{mM} \mathrm{KCl}, 12 \mathrm{mM}$ HEPES (pH 7.9), 5 $\mathrm{mM} \mathrm{MgCl}_{2}, 0.5 \mathrm{M}$ dithiothreitol, $0.5 \mathrm{mM}$ EDTA, $6 \%$ glycerol, $0.3 \mathrm{mM}$ phenylmethylsulfonyl fluoride, and $2 \mu \mathrm{g}$ of poly $(\mathrm{dI}-\mathrm{dC})$. The reaction mixture was incubated on ice for
$30 \mathrm{~min}$ and applied to a $4 \%$ nondenatured polyacrylamide gel in $0.02 \mathrm{M}$ Tris- $0.02 \mathrm{M}$ boric acid- $0.05 \mathrm{mM}$ EDTA. DNAprotein complexes and free DNA were located by autoradiography.

DNase I footprint assays. The binding sites of nuclear protein factors on DNA sequence elements were determined by DNase I footprint assays (44). End-labeled DNA and nuclear extracts were mixed in the buffer used for mobility shift assays and treated with freshly diluted DNase I for 1 min on ice. After extraction with phenol and chloroform, the DNA was applied to a $5 \%$ polyacrylamide gel in $8 \mathrm{M}$ urea. After electrophoresis, the DNA bands were visualized by autoradiography.

Nuclear run-on transcription assay. For the nuclear run-on transcription assay, nuclei were prepared from S-phase, non-S-phase, and cycloheximide-treated S-phase cells as described above. To obtain cycloheximide-treated non-Sphase cells, we treated cells with aphidicolin for $24 \mathrm{~h}$ and added cycloheximide $(20 \mu \mathrm{g} / \mathrm{ml})$ to the same medium $2 \mathrm{~h}$ before harvesting the cells. Nascent RNA transcripts were elongated by incubation of nuclei with labeled nucleotides as described previously (19). The labeled transcripts were isolated and hybridized with appropriate DNAs $(2 \mu \mathrm{g})$ fixed to nitrocellulose filters (23).

\section{RESULTS}

CCAAT and octamer elements are SRE of TH2B gene. In a previous study, we demonstrated that the cloned testisspecific rat TH2B gene has a promoter activity for $S$ phase-dependent transcription when introduced into several somatic cell lines (21; I. Hwang, unpublished results). Experiments with deletion mutations of the gene fused between the TH2B 5' upstream sequence (554 bp from immediately upstream of AUG codon) and CAT gene (pTH2BCAT) showed that two regions are involved in the S-phase-dependent transcription (21). One of the regions (between -153 and -109 bp from the transcription initiation site) contains two CCAAT elements, and the other region (between -109 and $-84 \mathrm{bp}$ ) contains the conserved octamer sequence element (the sequence is shown in Fig. 1A). Each region alone produces about half of the full increase (six- to sevenfold) of CAT activity during the $S$ phase (21). CCAAT and octamer (ATTTGCAT) elements have been shown to play important roles in S-phase-specific transcription of a human thymidine kinase (24) and a human somatic histone H2B (27) gene, respectively. The regions important for the S-phase-dependent transcription of the TH2B gene show remarkably similar sequence organization to a human somatic $\mathrm{H} 2 \mathrm{~B}$ regulatory region (27) (Fig. 1B).

A recent experiment showed that the TH2B-CAT with a deletion up to -140 bp still produces a full increase of CAT activity during the $S$ phase (data not shown). In this mutant, only one CCAAT element is present (at $-127 \mathrm{bp}$ ) between $-140 \mathrm{bp}$ and the octamer element at $-93 \mathrm{bp}$. Thus, it is possible that the CCAAT element at $-127 \mathrm{bp}$ and the octamer element play important roles in the S-phase-dependent transcription of the TH2B gene. To test this possibility, we introduced mutations into these elements as well as into the CCAAT downstream of the octamer $(-70 \mathrm{bp}$; [Fig. 2A]). The effect of these mutations on the expression of CAT in the $S$ phase of $C 3 H 10 T 1 / 2$ cells was investigated by gene transfer experiments (Fig. 2). Mutations in the octamer element OM-3 (Fig. 2B, lanes 3 and 4) reduced the $S$ phase-dependent increase of CAT activity to about half of the maximal increase (six- to sevenfold) obtained with nor- 
(A)

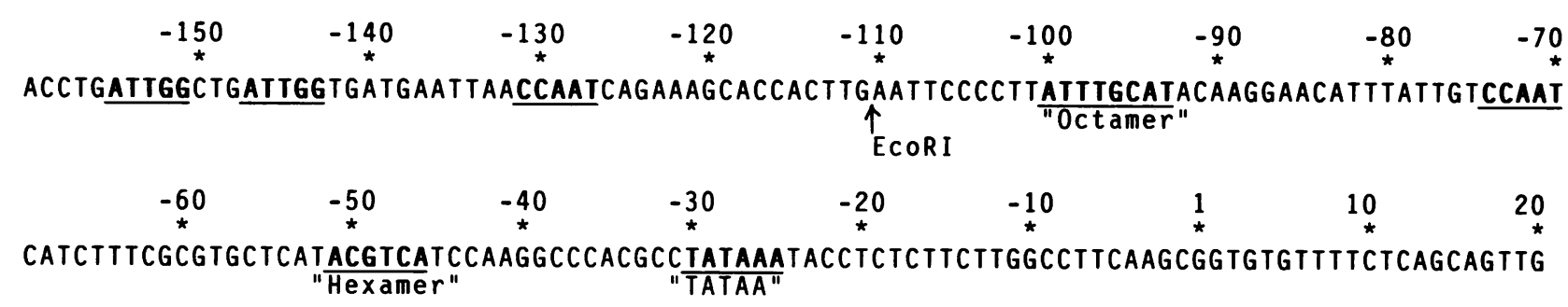

(B)

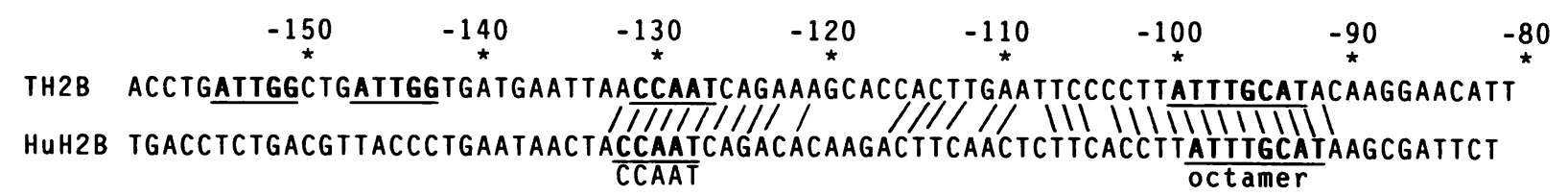

FIG. 1. Upstream sequence of the TH2B gene. (A) The 5' upstream sequence of TH2B gene is shown. The octamer and TATAA sequences are indicated, and CCAAT sequence elements are underlined. The EcoRI restriction site used in the insertion mutations is shown. The hexamer ACGTCA element found in plant histone genes (43) is also indicated. (B) The sequence within the regulatory region of TH2B gene was compared with the human H2B sequence. Octamer and CCAAT sequence elements are indicated, and sequences that are similar between the two genes are shown by connecting lines.

mal pTH2BCAT (lanes 1 and 2). Mutations in the CCAAT element at -127 bp (MACm; lanes 7 and 8), but not at -70 bp (MICm; lanes 5 and 6), reduced the S-phase-dependent increase. The pTH2BCAT containing mutations in both CCAAT elements $(\mathrm{BCm})$ showed a similar result to the mutations in the CCAAT at -127 bp (lanes 9 and 10). The results suggest that the CCAAT element at -127 bp alone may function as an SRE without the octamer element and that the CCAAT and octamer elements may cooperate in producing maximal stimulation of the S-phase transcription. A similar conclusion was drawn from the results obtained with deletion mutants in a previous study (21). The mutations in the CCAAT and octamer elements also reduced the production of CAT in the non-S phase as well as in the $S$ phase of the cell cycle. It is interesting that the downstream CCAAT element has no apparent effect on the S-phasedependent production of CAT.

Protein-binding sites within the minimal promoter region. The CCAAT and octamer elements are most probably the binding sites of the regulatory proteins which stimulate gene transcription during the $S$ phase. The mutations in these elements may prevent binding of the regulatory proteins. To investigate these possibilities, we mapped the proteinbinding sites on the TH2B 5'-flanking region by a DNase footprinting assay (44). The DNA fragment corresponding to -485 and +64 bp was labeled at the +64-bp end of the noncoding bottom strand with ${ }^{32} \mathrm{P}$ and incubated with nuclear extracts prepared from S-phase and non-S-phase cells. The mixtures were digested with a minimal amount of DNase I, and the DNA samples were resolved by electrophoresis on a high-resolution polyacrylamide gel (Fig. 3). The results show that at least five sites are involved in protein binding within the minimal promoter region involved in the S-phase-dependent transcription $(-140 \mathrm{bp}$ from the transcription initiation site). The protein-binding sites are at between -23 and -30 bp containing TATAA (site E), between -39 and $-57 \mathrm{bp}$ (site D), between -62 and $-76 \mathrm{bp}$ containing CCAAT (site C), between -87 and -98 bp

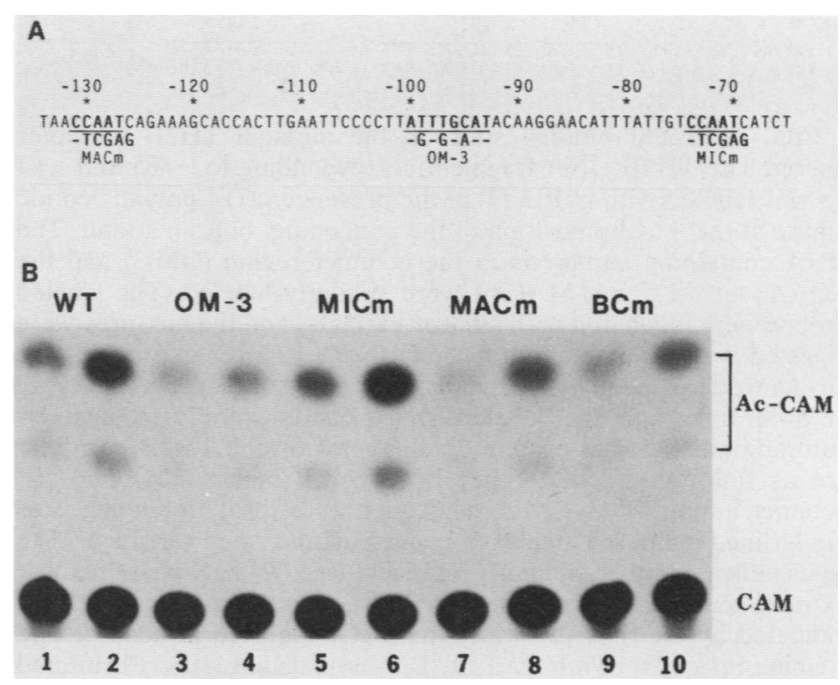

FIG. 2. Effect of mutations in the octamer and CCAAT elements on the S-phase-dependent transcription. The octamer (OM-3) and the CCAAT mutants (MACm, MICm, and BCm) of pTH2BCAT were cotransfected with pRSV $\beta$-gal into $C 3 H$ 10T1/2 cells, and the cells were synchronized by incubation with aphidicolin for $24 \mathrm{~h}$ as described in Materials and Methods. After release from the aphidicolin block the cells were harvested, and $\beta$-galactosidase activity was determined as described in Materials and Methods. The amounts of extracts containing the same $\beta$-galactosidase activity were used for the assay of CAT activity to normalize different transfection efficiencies among different culture dishes. Non-Sphase cells were incubated with aphidicolin for $31 \mathrm{~h}$ and harvested. (A) Sequences of octamer and CCAAT mutants. The octamer and CCAAT elements are underlined. The changed bases are indicated by bold letters underneath. The mutant $\mathrm{BCm}$ contains mutations in both CCAAT elements. (B) Lanes: 1 and 2, TH2B-CAT; 3 and 4, OM-3; 5 and 6, MICm; 7 and $8, \mathrm{MACm} ; 9$ and $10, \mathrm{BCm}$. The odd numbers represent non-S-phase cells, and the even numbers represent S-phase cells. Ac-CAM, Acetoxychloramphenicol. 


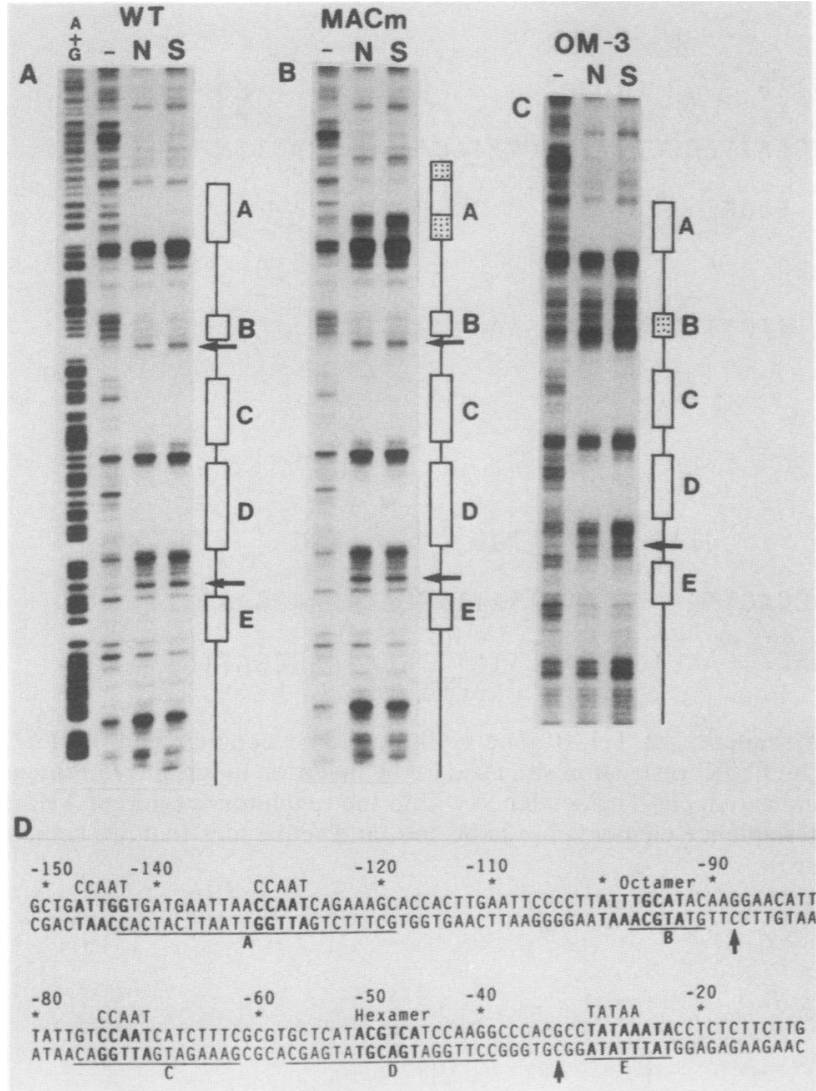

FIG. 3. Protein-binding sites on the minimal TH2B promoter region. The TH2B DNA fragment corresponding to -485 and +64 bp was labeled with $\left.{ }^{32} \mathrm{P}\right]$ ATP in the presence of T4 polynucleotide kinase at the +64-bp position of the noncoding, bottom strand. The DNA containing mutations in the octamer region (OM-3) and the CCAAT at -127 bp (MACm) were similarly labeled. The labeled probes were incubated with nuclear extracts, and the mixtures were digested with a minimal amount of DNase I. After extraction, the DNAs were resolved by electrophoresis on a $5 \%$ polyacrylamide gel in $8 \mathrm{M}$ urea and the labeled DNA bands were visualized by autoradiography. The protein-binding sites on the TH2B promoter are as follows: A, wild type; B, CCAAT mutant (MACm); C, octamer mutant (OM-3); D, the sequences of protein-binding sites (underlined) and the nuclease-hypersensitive sites (arrows). The lanes indicated by,$- \mathrm{S}$, and $\mathrm{N}$ represent free DNA, S-phase nuclear extract, and non-S-phase nuclear extract, respectively. The lane indicated by $A+G$ is the wild-type probe digested at adenine and guanine residues. Symbols: $\square$, DNase-resistant sites; $\left[\begin{array}{l}0 \\ \text {, altered }\end{array}\right.$ protein binding in mutants; $\longleftarrow$, nuclease-hypersensitive sites.

containing octamer (site B), and between -119 and -143 bp containing CCAAT (site A) (Fig. 3A and D). It is interesting that a cytosine downstream of the octamer element is hypersensitive to DNase I (Fig. 3A and D, arrow). The region containing two CCAAT elements (at -142 and -150 bp) also appears to be bound by proteins. Region $D$ contains a hexamer element, ACGTCA, which is present in several plant histone genes and is recognized by a protein of 36,000 daltons (43). No protein binds to the mutated octamer, OM-3 (Fig. 3C). The mutations in CCAAT at -127 bp (MACm) changed the pattern of the DNase sensitivity of the upstream region, suggesting that the proteins binding to the upstream CCAAT elements were affected by the mutations at the -127-bp CCAAT (Fig. 3B). The results shown in Fig. 3 suggest that several different proteins interact with the minimal $\mathrm{TH} 2 \mathrm{~B}$ promoter and the action of CCAAT and octamer elements are most probably mediated by the proteins which bind to these elements. The proteins which bind to these regulatory elements are also present in non-S-phase cells. The significance of this result is discussed further below.

Proper alignment of CCAAT and the octamer element is required for the maximal activation of the S-phase-specific transcription. A possible interaction between the proteins binding to the CCAAT element at $-127 \mathrm{bp}$ and the octamer element for the maximal S-phase-specific transcription was investigated by introducing various lengths of DNA (such as $4,11,17,23,26$, and 32 bp) between the CCAAT and octamer sequence elements to generate half and integral turns of DNA helix (Fig. 4A). The resulting DNAs were introduced into $\mathrm{C} 3 \mathrm{H} 10 \mathrm{~T} 1 / 2$ cells, and CAT activity was determined. The S-phase-dependent production of CAT is strongly dependent on spacings between the CCAAT and octamer elements, with apparent 10 -bp periodicity (Fig. 4B and $\mathrm{C})$. When the insertions were integral or near-integral turns of the helix such as 11,23 , and $32 \mathrm{bp}$, the $\mathrm{S}$ phase-dependent production of CAT was increased about sevenfold. Mutants with half turns of the helix (4- and 17 bp-insertions) showed a threefold increase of transcription in the $\mathrm{S}$ phase. However, when the half-turn insertions became longer (for example, $26 \mathrm{bp}$ ), the S-phase dependency did not diminish as much as the reduction obtained with shorter half-turn insertions (Fig. 4B, lanes 11 and 12). This indicates that the requirement of the proper alignment of the two elements is less stringent when insertion is longer, probably owing to the flexibility of DNA. The results obtained with the insertion mutants most probably reflect the interaction of the proteins binding to the CCAAT element at -127 bp and the octamer element, but not to any other elements. This conclusion is based on the following results: (i) the reduction of S-phase-dependent production of CAT from TH2B-CAT with the mutations in the CCAAT (MACm) and octamer elements (Fig. 2); (ii) the absence of S-phase-dependent transcription from the -84 -bp deletion mutants (21) or from the downstream CCAAT mutant (MICm), thus suggesting that the downstream elements are not involved in the $S$ phase-dependent transcription; and (iii) the absence of any effect on the production of CAT when a 4-bp DNA fragment was inserted between the CCAAT and mutant octamer in plasmid OM-3 (data not shown). The insertions also had no effect on the binding of the proteins to the CCAAT and wild-type octamer elements (data not shown). The result was obtained by mobility shift assays with the DNA fragments containing the CCAAT and octamer elements which were isolated from wild-type and insertion mutant plasmids.

S-phase-dependent transcription of the TH2B gene does not require synthesis of new proteins. Others have reported that the nuclear protein factors interacting with SRE of human thymidine kinase (24) and hamster histone $\mathrm{H} 3.2$ (2) genes are increased during the $S$ phase of the cell cycle. On the other hand, the factors interacting with the regulatory sequences of human $\mathrm{H} 3$ and $\mathrm{H} 4$ genes persist throughout the cell cycle $(35,45)$. To determine whether the regulatory proteins for TH2B SRE are induced during the $S$ phase of the cell cycle, we investigated the binding of proteins to various elements in the TH2B upstream region by using the nuclear extracts prepared from S-phase cells (released for 2 to $3 \mathrm{~h}$ from the aphidicolin block) and non-S-phase cells (maintained with aphidicolin for $24 \mathrm{~h}$; G1/S border). S-phase and non-S-phase nuclear extracts produced the same protein-binding patterns (Fig. 3). The proteins which bind to the CCAAT and octamer 

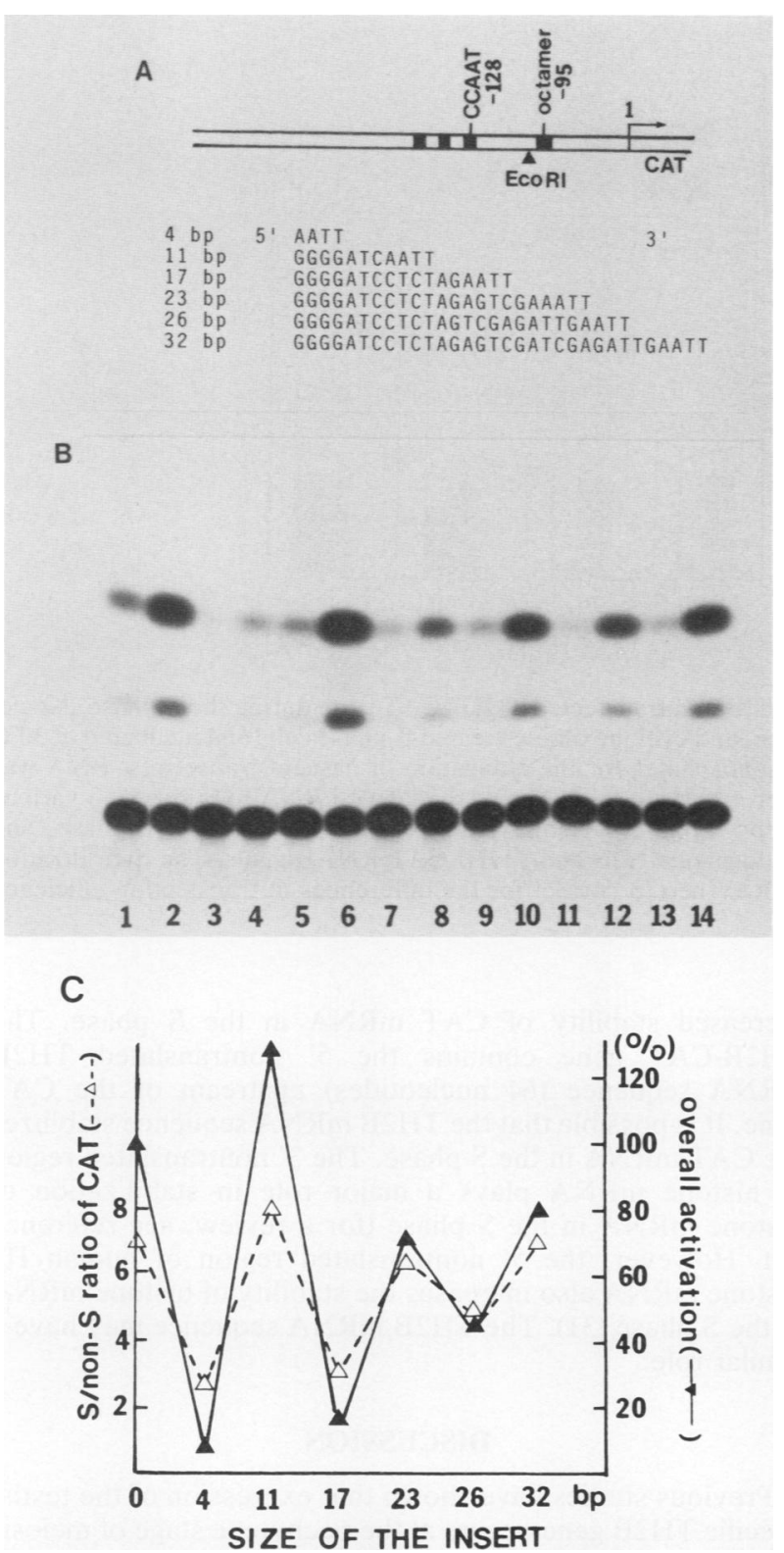

FIG. 4. Cooperative interaction between the CCAAT and octamer elements for the S-phase-dependent transcription. The insertion mutants and a control DNA (pRSV $\beta$-gal) were cotransfected into the cells, and the effect of the insertions on the S-phasedependent CAT activity as well as on the overall activation of the CAT gene was tested. After transfection, the cells were synchronized with aphidicolin for $24 \mathrm{~h}$ and released from the aphidicolin block for $7 \mathrm{~h}$. (A) The sequences of inserts and the insertion site within the pTH2BCAT (the EcoRI site indicated). The CCAAT and octamer sequence elements are indicated. (B) CAT activities of insertion mutants. Lanes: 1 and 2, no insertion (pTH2BCAT); 3 and 4, 4-bp insertion; 5 and 6, 11-bp insertion; 7 and 8, 17-bp insertion; 9 and 10, 23-bp insertion; 11 and 12, 26-bp insertion; 13 and 14, 32-bp insertion. The odd numbers for each insertion mutant represent the non-S-phase cells, and the even numbers represent the S-phase cells. (C) The ratios of S-phase to non-S-phase CAT activities and the overall activation (CAT activities of $S$ phase cells). Acetylated chloramphenicol spots were scraped from the thin-layer plates, and the radioactivity was determined in a scintillation counter. Symbols: $\Delta$, ratio of S-phase to non-S-phase values; $\boldsymbol{\Delta}$, overall activation. Data are expressed as percentage of the S-phase CAT activity of the wild-type TH2B-CAT fusion gene.

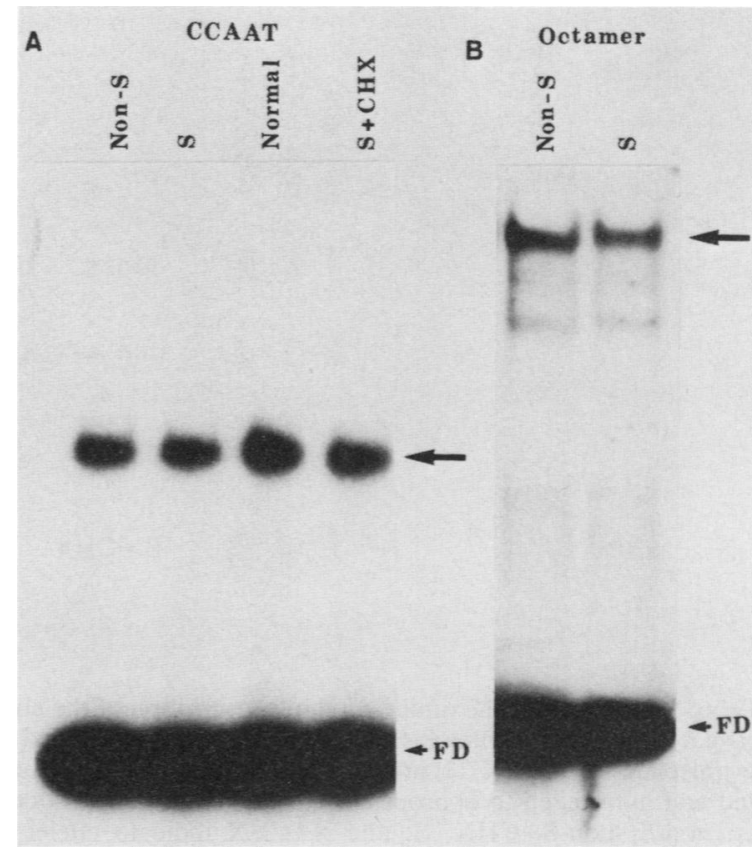

FIG. 5. The presence of the CCAAT and octamer-binding factors throughout the cell cycle. (A) Nuclear extracts were prepared from non-S-phase, S-phase, cycloheximide-treated S-phase, and actively dividing normal cells as described in Materials and Methods. The labeled $44-b p$ probe $(-153$ to $-110 \mathrm{bp})$ containing the CCAAT element at -127 bp was incubated with four different nuclear extracts on ice and applied to a nondenatured polyacrylamide gel. Non-S, S, Normal, and S+CHX indicate the nuclear extracts prepared from non-S-phase, S-phase, normal, and cycloheximide-treated S-phase cells, respectively. The arrow indicates the DNA-protein complex; FD indicates free DNA. (B) The octamer-binding proteins in S-phase and non-S-phase nuclear extracts. A double-stranded oligonucleotide corresponding to -108 and -84 bp from the transcription initiation site was used as a probe.

elements are present in both S-phase and non-S-phase cells (Fig. 3). The possible changes in the level of CCAAT and octamer-binding proteins under various conditions were investigated by a DNA mobility shift assay. A labeled probe containing the CCAAT element $(-109$ to $-153 \mathrm{bp})$ and nuclear extracts from S-phase and non-S-phase cells were mixed, and the formation of a DNA-protein complex was assayed by retardation of the mobility of labeled DNA. The results showed that the amount of DNA-protein complex formed was the same with the S-phase and non-S-phase nuclear extracts (Fig. 5A). Also, the presence of cycloheximide during the release of cells from the aphidicolin block had no apparent effect on the level of CCAAT-specific protein (Fig. 5A, lane $\mathrm{S}+\mathrm{CHX}$ ). The level of octamerbinding proteins was also determined by a mobility shift assay with a synthetic oligonucleotide corresponding to the -80 - and $-110-b p$ region of the TH2B promoter (Fig. 5B). The amount of octamer-binding protein is also the same in the non-S-phase and S-phase nuclear extracts. Thus, it appears that the nuclear protein factors involved in the S-phase-dependent increase of transcription are already present before the cells enter the $S$ phase. The synchrony of confluence-arrested or semiconfluent cells with aphidicolin treatment showed the same results (data not shown).

It is possible that the S-phase-dependent increase of TH2B gene transcription is achieved by interaction between the DNA-binding regulatory proteins with other non-DNA-bind- 

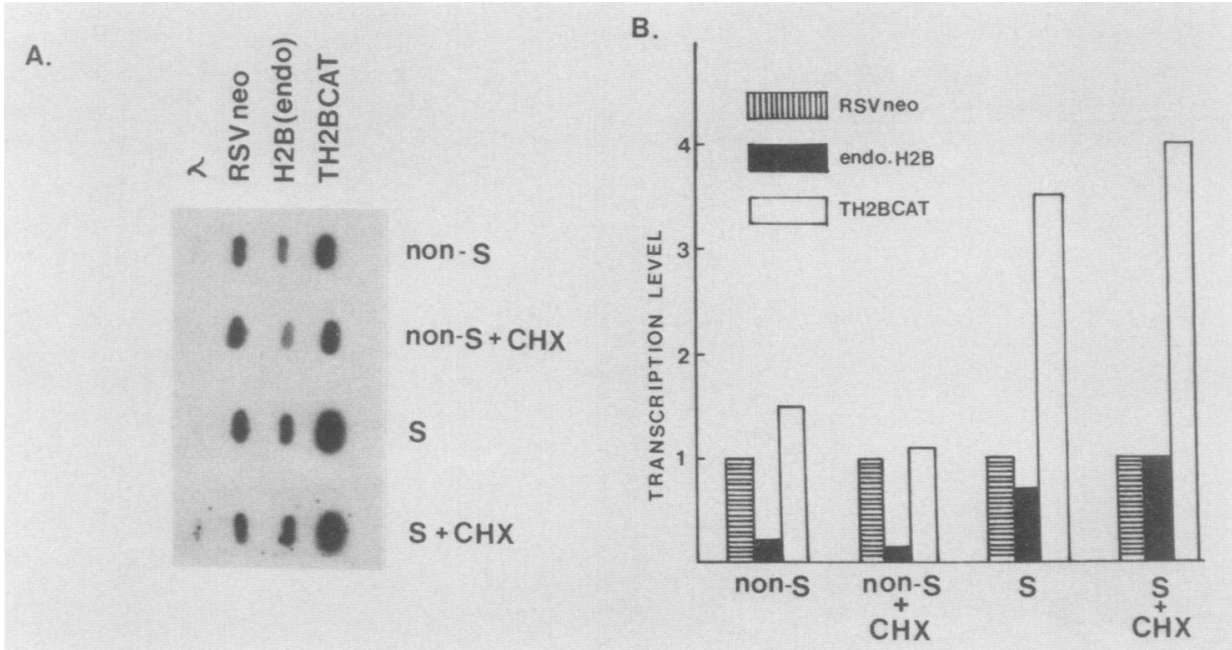

FIG. 6. Effect of cycloheximide on the transcription of the endogenous H2B and transfected TH2B-CAT gene during the S phase. Nuclei were isolated from non-S-phase, S-phase, cycloheximide-treated non-S-phase, and cycloheximide-treated S-phase cells and incubated at $30^{\circ} \mathrm{C}$ in the presence of $\left[\alpha-{ }^{32} \mathrm{P}\right] \mathrm{GTP}$ and three other unlabeled ribonucleoside triphosphates for the elongation of nascent transcripts. RNA was isolated and hybridized to appropriate DNAs bound to a nitrocellulose filter. (A) Autoradiogram of the labeled RNA hybridized to various DNAs. non-S, non-S $+\mathrm{CHX}, \mathrm{S}$, and $\mathrm{S}+\mathrm{CHX}$ indicate nuclei from the non-S-phase, cycloheximide-treated non-S-phase, S-phase, and cycloheximide-treated S-phase cells, respectively. (B) Intensities of the endogenous H2B and TH2B-CAT RNA signals. The hybridization signals were quantitated by densitometry and normalized by the signal of RSV-neo to correct for the differences in transfection efficiency among different dishes.

ing nuclear proteins which are induced during the $S$ phase. To investigate this possibility, we investigated the effect of cycloheximide on the transcription of the TH2B-CAT fusion gene during the release of aphidicolin-blocked cells by a nuclear run-on transcription assay. C3H 10T1/2 cells were cotransfected with the chimeric TH2B-CAT and $R S V$-neo (16) genes and synchronized by using the aphidicolin block protocol. The cells were released from the block for $2 \mathrm{~h}$ in the presence of cycloheximide to inhibit protein synthesis during the $\mathrm{S}$ phase, and their nuclei were isolated. Nuclear run-on transcription was carried out in the presence of labeled nucleotides. The labeled RNA was hybridized to various DNAs fixed on a nitrocellulose filter. The results are shown in Fig. 6. The intensities of hybridization signals were compared with that of the neomycin gene transcript, and the ratios for the nuclei from aphidicolin-treated cells (non-S) and the nuclei from the cells released from the aphidicolin block in the presence (S+CHX) and absence (S) of cycloheximide were compared (Fig. 6B). The hybridization signals were normalized with those of the neomycin RNA to correct for the differences in the transfection efficiency among different culture dishes. The levels of transcription of the TH2B-CAT fusion gene in the S-phase and cycloheximide-treated S-phase nuclei were both increased about fourfold compared with that of the non-S-phase nuclei. Therefore, cycloheximide does not have any effect on the $S$ phase-specific transcription of the TH2B-CAT fusion gene during the $S$ phase. Also, the endogenous mouse $H 2 B$ gene transcription was unaffected by the cycloheximide treatment (Fig. 6B). This result strongly suggests that synthesis of new proteins is not required for the increase in the transcription of TH2B and the endogenous mouse H2B genes upon entry of the cells into the $S$ phase of the cell cycle. The degree of stimulation of the S-phase-dependent transcription in isolated nuclei was about threefold, in contrast to the six- to sevenfold stimulation of CAT protein production from the TH2B-CAT gene. This discrepancy could be due to the increased stability of CAT mRNA in the $S$ phase. The TH2B-CAT gene contains the $5^{\prime}$ nontranslated TH2B mRNA sequence (64 nucleotides) upstream of the CAT gene. It is possible that the TH2B mRNA sequence stabilizes the CAT mRNA in the $S$ phase. The $3^{\prime}$ nontranslated region of histone mRNA plays a major role in stabilization of histone mRNA in the $S$ phase (for a review, see reference 39). However, the $5^{\prime}$ nontranslated region of human $\mathrm{H} 3$ histone mRNA also increases the stability of histone mRNA in the $S$ phase (31). The TH2B mRNA sequence may have a similar role.

\section{DISCUSSION}

Previous studies have shown that expression of the testisspecific TH2B gene occurs at the pachytene stage of meiosis I during spermatogenesis in rat testis (23) and that synthesis of TH2B histones is independent of DNA replication $(3,28)$. Surprisingly, the cloned TH2B gene is regulated in an $\mathrm{S}$-phase-dependent manner in the same way as somatic $\mathrm{H} 2 \mathrm{~B}$ gene when introduced into somatic cells (21). Also, the upstream region directs the transcription of a reporter gene in an S-phase-dependent manner in mouse embryo fibroblast cells as well as in other cells such as rat thyroid and choriocarcinoma cells (21; I. Hwang, unpublished results). DNase footprint analysis suggests that the expression of the TH2B gene requires the interaction of proteins with multiple sites in the TH2B promoter region. However, two regions appear to play key roles in the maximal stimulation of the TH2B gene during the $S$ phase. In this study we determined, by analysis of the TH2B-CAT gene containing mutations in the CCAAT and octamer elements, that the SRE in the two regions are the CCAAT element at $-127 \mathrm{bp}$ and the octamer motif at $-93 \mathrm{bp}$ from the transcription initiation site. The two elements appear to be important for the overall transcriptional activity of the TH2B gene in the $S$ phase and the non-S phase of the cell cycle as well. The two elements can 
independently increase the S-phase-dependent transcription. However, each produces only half of the maximal increase achieved by both elements. Experiments with insertion mutants show that the factors interacting with the two elements most probably interact with each other to achieve the maximal increase. When the DNA helix rotates one-half turn to place the two elements at opposite side of the DNA, the S-phase-dependent increase is reduced to half the original value. The level of protein binding to each site appears to be unaffected by the insertion of space between them (data not shown). Cooperative interactions of regulatory proteins are important for regulation of many genes $(5,38)$. It was also reported that the octamer element involved in tissuespecific transcription of the immunoglobulin gene mediates the action of upstream enhancers (34). CCAAT and octamer elements are also involved in the S-phase-dependent transcription of human thymidine kinase (24) and H2B (27) genes, respectively. The nature of the proteins binding to the TH2B CCAAT and octamer element is not known. It is quite likely that a protein similar to OTF-1, the 90-kilodalton protein specific for the human H2B octamer element (12), also recognizes TH2B octamer. OTF-1 also binds to the adenovirus DNA replication origin (32). Therefore, there is a good possibility that OTF-1 activates both DNA replication and histone gene transcription at the same time during the $S$ phase. There are many different CCAAT-binding proteins in the cell $(4,6,10,36)$. CCAAT and the surrounding sequences may control the type of protein binding to the DNA. It is possible that there is a CCAAT-binding protein which stimulates the S-phase-dependent gene transcription.

The sequence organization of the TH2B upstream region containing the two SRE is very similar to that of a human somatic $\mathrm{H} 2 \mathrm{~B}$ gene (27; Fig. 1B). In the human $\mathrm{H} 2 \mathrm{~B}$ gene, the octamer motif alone is sufficient for the S-phase-dependent increase of transcription and the upstream sequence, including a CCAAT element, is involved in the overall increase of transcriptional activity of the gene in the $S$ phase and the non-S phase of the cell cycle (27). A rat somatic H2B gene also has similar sequence organization to the TH2B and human H2B upstream regions. Preliminary experiments suggest that only the octamer element functions as the SRE in the rat $\mathrm{H} 2 \mathrm{~B}$ gene as in the human $\mathrm{H} 2 \mathrm{~B}$ gene (Y.-C. Choi, unpublished data). The significance of this difference between the testis-specific and DNA-replication-independent TH2B and somatic H2B genes is not clear.

It has been reported that the level of proteins interacting with SRE of a human thymidine kinase (24) and a hamster histone H3 (2) gene is increased during the $S$ phase. This suggests the possibility that the $S$-phase-dependent increase of histone gene transcription is achieved by induction of synthesis of gene regulatory proteins during the initiation of the $\mathrm{S}$ phase. However, the TH2B and mouse endogenous $\mathrm{H} 2 \mathrm{~B}$ genes do not appear to require new protein synthesis for S-phase-dependent transcription. Treatment of the cells with cycloheximide during the initiation of the $S$ phase does not affect the in vivo transcription of TH2B or endogenous mouse $\mathrm{H} 2 \mathrm{~B}$ genes, as demonstrated by a nuclear run-on transcription assay. Furthermore, the level of the protein factors binding to the CCAAT and octamer elements, as well as other regions, is not affected during the cell cycle or by the presence of cycloheximide during the $S$ phase. Therefore, posttranscriptional modification of the regulatory factors may be involved in the S-phase-dependent transcription of $\mathrm{H} 2 \mathrm{~B}$ genes. In the drug-induced cell synchrony, the cells accumulate at the G1/S border before entering the $S$ phase. Therefore, it is possible that regulatory factors are produced at the G1/S border. However, the transcription in early $S$ phase is still not affected by cycloheximide, suggesting that posttranscriptional modification appears to be important for the S-phase-dependent increase of $\mathrm{H} 2 \mathrm{~B}$ gene transcription in this artificially induced synchronized cell system. OTF-1 and the regulatory proteins for other histone SRE are present in pre-S-phase cells and are not affected by cell cycle. An example of posttranslational modification of preexisting gene regulatory proteins as a mechanism of gene activation can be found in the genes activated by cyclic AMP (30). The factors regulating human $\mathrm{H} 3$ and $\mathrm{H} 4$ histone genes also persist during the cell cycle $(35,45)$.

The presence of the DNA sequence elements involved in gene regulation in an S-phase-dependent manner in the testis-specific and DNA replication-independent TH2B gene is surprising. The biological significance of the maintenance of SRE during evolution is not clear. Since the SRE of somatic histones are not cell type specific, it is possible that the TH2B SRE are repressed in somatic cells in the rat. Preliminary experiments with nuclear extracts showed that various tissues, including testis, show the same DNasefootprinting pattern as the pattern obtained with the mouse fibroblast nuclear extracts (K. Lim, unpublished results). However, the TH2B promoter region is methylated at several sites in various tissues but not in testis tissue (Y.-C. Choi, unpublished results). The methylated bases could prevent binding of one or more of the regulatory proteins to the TH2B promoter. These results suggest that the TH2B SRE could be accessible for binding to the nuclear regulatory factors only in pachytene spermatocytes. These possibilities are being investigated in our laboratory.

\section{ACKNOWLEDGMENTS}

We thank William J. Rutter for pRSV $\beta$-gal. Aphidicolin was generously supplied by the Natural Products Branch, National Cancer Institute.

K.L. was supported in part by a fellowship from the Korean Science and Engineering Foundation. This work was supported by Public Health Service grant HD-20136 from the National Institute of Child Health and Human Development.

\section{LITERATURE CITED}

1. An, G., K. Hidaka, and L. Siminovitch. 1982. Expression of bacterial $\beta$-galactosidase in animal cells. Mol. Cell. Biol. 2: 1628-1632.

2. Artishevsky, A., S. Wooden, A. Sharma, E. Resendez, Jr., and A. S. Lee. 1987. Cell-cycle regulatory sequences in a hamster histone promoter and their interactions with cellular factors. Nature (London) 328:823-827.

3. Chiu, M., and J. L. Irvin. 1985. Effect of inhibition of DNA synthesis on histone synthesis, turnover, and deposition in the rat testis. Arch. Biochem. Biophys. 236:260-265.

4. Chodosh, L. A., A. S. Baldwin, R. W. Carthew, and P. A. Sharp. 1988. Human CCAAT-binding proteins have heterologous subunits. Cell 53:11-24.

5. Cohen, R., and M. Meselson. 1988. Periodic interactions of heat shock transcriptional elements. Nature (London) 332:856-858.

6. Cohen, R. S., M. Sheffery, and C. G. Kim. 1986. Partial purification of a nuclear protein that binds to the CCAAT box of the mouse $\alpha$-globin gene. Mol. Cell. Biol. 6:821-832.

7. Cordeiro-Stone, M., and D. G. Kaufman. 1984. Kinetics of DNA replication in $\mathrm{C} 3 \mathrm{H} 10 \mathrm{~T} 1 / 2$ cells synchronized by aphidicolin. Biochemistry 24:4815-4822.

8. Dalton, S., and J. R. E. Wells. 1988. A gene-specific promoter element is required for optimal expression of the histone $\mathrm{H} 1$ gene in S-phase. EMBO J. 7:49-56.

9. Dignam, J. D., R. M. Lebovitz, and R. G. Roeder. 1983 Accurate transcription initiation by RNA polymerase II in a soluble extract from isolated mammalian nuclei. Nucleic Acids 
Res. 11:1475-1489.

10. Dorn, A., J. Bollekens, A. Staub, C. Beboist, and D. Mathis. 1987. A multiplicity of CCAAT box-binding proteins. Cell 50:863-872.

11. Edlund, T., M. D. Walker, P. J. Barr, and W. J. Rutter. 1985. Cell-specific expression of the rat insulin gene: evidence for role of two distinct 5 ' flanking elements. Science 230:912-916.

12. Fletcher, C., N. Heintz, and R. G. Roeder. 1987. Purification and characterization of OTF-1, a transcription factor regulating cell cycle expression of a human $\mathrm{H} 2 \mathrm{~b}$ gene. Cell 51:773-781.

13. Fried, M., and D. M. Crothers. 1981. Equilibria and kinetics of lac repressor-operator interactions by polyacrylamide gel electrophoresis. Nucleic Acids Res. 9:6505-6525.

14. Garner, M. M., and A. Revzin. 1981. A gel electrophoresis method for quantifying the binding of proteins to specific DNA regions: application to components of the Escherichia coli lactose operon regulatory system. Nucleic Acids Res. 9:30473060.

15. Gorman, C. 1985. High efficiency gene transfer into mammalian cells, p. 143-190. In D. M. Glover (ed.), DNA cloning: a practical approach, vol. 2. IRL Press, Washington, D.C.

16. Gorman, C., P. Padmannabhan, and B. H. Howard. 1983. High efficiency DNA-mediated transformation of primate cells. Science 221:551-553.

17. Gorman, C. M., L. F. Moffat, and B. H. Howard. 1982. Recombinant genomes which express chloramphenicol acetyltransferase in mammalian cells. Mol. Cell. Biol. 2:1044-1051.

18. Green, L., I. Schlaffer, K. Wright, M. L. Moreno, D. Berand, G. Hafer, J. Stein, and G. Stein. 1986. Cell cycle-dependent expression of a stable episomal human histone gene in a mouse cell. Proc. Natl. Acad. Sci. USA 83:2315-2319.

19. Groudine, M., M. Peretz, and H. Weintraub. 1981. Transcriptional regulation of hemoglobin switching in chicken embryos. Mol. Cell. Biol. 1:281-288.

20. Heintz, N., H. L. Sive, and R. Roeder. 1983. Regulation of human histone gene expression: kinetics of accumulation and changes in the rate of synthesis and in half-lives of individual histone mRNAs during HeLa cell cycle. Mol. Cell. Biol. 3: 539-550.

21. Hwang, I., and C.-B. Chae. 1989. S-phase-specific transcription regulatory elements are present in a replication-independent testis-specific H2B histone gene. Mol. Cell. Biol. 9:1005-1013.

22. Kalb, V. F., Jr., and R. W. Bernlohr. 1977. A new spectrophotometric assay for protein in cell extracts. Anal. Biochem. 82:362-371.

23. Kim, Y.-J., I. Hwang, L. L. Tres, A. L. Kierszenbaum, and C.-B. Chae. 1987. Molecular cloning and differential expression of somatic and testis-specific H2B histone genes during rat spermatogenesis. Dev. Biol. 124:23-34.

24. Knight, G. B., J. M. Gudas, and A. B. Pardee. 1987. Cellcycle-specific interaction of nuclear DNA-binding proteins with a CCAAT sequence from the human thymidine kinase gene. Proc. Natl. Acad. Sci. USA 84:8350-8354.

25. Kroeger, P., C. Stewart, T. Schaap, A. van Wijnen, J. Hirshman, S. Helms, G. Stein, and J. Stein. 1987. Proximal and distal regulatory elements that influence in vivo expression of a cell cycle-dependent human $\mathrm{H} 4$ histone gene. Proc. Natl. Acad. Sci. USA 84:3982-3986.

26. Kunkel, T. 1985. Rapid and efficient site-specific mutagenesis without phenotypic selection. Proc. Natl. Acad. Sci. USA 82:488-492.

27. LaBella, F., H. L. Sive, R. G. Roeder, and N. Heintz. 1988. Cell-cycle regulation of a human histone $\mathrm{H} 2 \mathrm{~b}$ gene is mediated by the H2b subtype-specific consensus element. Genes Dev.
2:32-39.

28. Meistrich, M. L. 1987. Proteins of the meiotic cell nucleus, p. 333-353. In P. B. Moens (ed.), Meiosis. Academic Press, Inc., New York.

29. Meistrich, M. L., B. O. Reid, and W. J. Barcellona. 1975. Meiotic DNA synthesis during mouse spermatogenesis. J. Cell Biol. 64:211-222.

30. Montminy, M., and L. M. Bilezikjian. 1987. Binding of a nuclear protein to the cyclic-AMP response element of the somatostatin gene. Nature (London) 328:175-178.

31. Morris, T., F. Marashi, L. Weber, E. Hickey, D. Greenspan, J. Bonner, J. Stein, and G. Stein. 1986. Involvement of the 5 -leader sequence in coupling the stability of a human $\mathrm{H} 3$ histone mRNA with DNA replication. Proc. Natl. Acad. Sci. USA 83:981-985.

32. O'Neill, E. A., C. Fletcher, C. R. Burrow, N. Heintz, R. G. Roeder, and T. J. Kelly. 1988. Transcription factor OTF-1 is functionally identical to the DNA replication factor NF-III. Science 241:1210-1213.

33. Osley, M. A., J. Gould, S. Kim, M. Kane, and L. Hereford. 1986. Identification of sequences in a yeast histone promoter involved in periodic transcription. Cell 45:537-544.

34. Panslow, T. J., S. D. Jones, B. Bond, and K. R. Yamamoto. 1987. The immunoglobulin octanucleotide: independent activity and selective interaction with enhancers. Science 235:1498-1987.

35. Pauli, U., S. Chrysogelos, G. Stein, J. Stein, and H. Nick. 1987. Protein-DNA interactions in vivo upstream of a cell cycleregulated human $\mathrm{H} 4$ histone gene. Science 236:1308-1311.

36. Raymondjean, M., S. Cereghini, and M. Yaniv. 1988. Several distinct "CCAAT" box binding proteins coexist in eukaryotic cells. Proc. Natl. Acad. Sci. USA 85:757-761.

37. Sanger, F., S. Nicklen, and A. R. Coulson. 1977. DNA sequencing with chain-terminating inhibitors. Proc. Natl. Acad. Sci. USA 74:5463-5468.

38. Schule, R., M. Muller, H. Otsuka-Murakami, and R. Renkawitz. 1988. Cooperativity of the glucocorticoid receptor and the CACCC-box binding factor. Nature (London) 332:87-90.

39. Schumperli, D. 1988. Multilevel regulation of replication-dependent histone genes. Trends Genet. 4:187-191.

40. Singh, H., R. Sen, D. Baltimore, and P. A. Sharp. 1986. A nuclear factor that binds to a conserved sequence motif in transcriptional control elements of immunoglobulin genes. Nature (London) 319:154-158.

41. Sittman, D. B., R. A. Graves, and W. F. Marzluff. 1983. Histone mRNA concentrations are regulated at the level of transcription and mRNA degradation. Proc. Natl. Acad. Sci. USA 80: 1849-1853.

42. Swiler-Tuyns, A., and B. M. Patterson. 1987. Cell cycle regulation of a mouse histone $\mathrm{H} 4$ gene requires the $\mathrm{H} 4$ promoter. Mol. Cell. Biol. 7:1048-1054.

43. Tabata, T., H. Takase, S. Takayama, K. Mikami, A. Nakatsuka, T. Kawata, T. Nakayama, and M. Iwabuchi. 1989. A protein that binds to a cis-acting element of wheat histone genes has a leucine zipper motif. Science 245:965-967.

44. Treisman, R. 1986. Identification of a protein-binding site that mediates transcriptional response of the $\mathrm{c}$-fos gene to serum factors. Cell 46:567-574.

45. van Wijnen, A. J., J. L. Stein, and G. S. Stein. 1987. A nuclear protein with affinity for the $5^{\prime}$ flanking region of a cell cycle dependent human $\mathrm{H} 4$ histone gene in vitro. Nucleic Acids Res. 15:1679-1698.

46. van Wijnen, A. J., K. L. Wright, R. F. Massung, M. Gerretsen, J. L. Stein, and G. S. Stein. 1988. Two target sites for protein binding in the promoter region of a cell cycle regulated human H1 histone gene. Nucleic Acids Res. 16:571-592. 